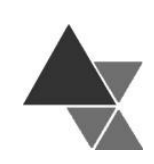

\title{
Insegurança Alimentar no Brasil após crise, sua evolução de 2004 a 2017-2018 e comparação com a variação da pobreza
}

\author{
Rodolfo Hoffmann ${ }^{1}$
}

Dados para aplicação da Escala Brasileira de Insegurança Alimentar (EBIA) foram coletados pelo Instituto Brasileiro de Geografia e Estatística (IBGE) em complementos da Pesquisa Nacional por Amostra de Domicílios (PNAD) em 2004, 2009 e 2013. Com a divulgação dos dados obtidos na Pesquisa de Orçamentos Familiares $2017-$ 2018, os pesquisadores passam a dispor de uma série de quatro anos de dados sobre o tema provenientes de amostras representativas do que ocorre em todo o território do país. A insegurança alimentar diminuiu de 2004 a 2013, mas cresceu em 2017-2018. Os dados indicam que a insegurança alimentar grave em 2017-2018 é maior do que em 2013, mas fica abaixo do valor observado em 2009. Mas a insegurança alimentar de qualquer grau em 2017 2018 supera até mesmo o valor observado em 2004. Analisando dados por Unidade da Federação mostra-se a forte associação da insegurança alimentar com a distribuição da renda e particularmente com medidas de pobreza. Verifica-se, com dados da PNAD, que o nível de pobreza caiu de 2003 a 2014 e, embora tenha subido de 2014 a 2017-2018, nestes anos fica substancialmente abaixo do valor observado em 2004. Argumenta-se, então, que o grande crescimento da insegurança alimentar leve de 2013 a 2017-2018 se deve, em parte, ao caráter parcialmente subjetivo da EBIA. Em outra seção do artigo, os microdados da POF 2017-2018 são utilizados para verificar, por meio de modelos de lógite, quais são os principais determinantes da probabilidade de insegurança alimentar em um domicílio. Comprovou-se que o aumento da renda e da escolaridade são instrumentos básicos para reduzir a insegurança alimentar e que rendas mais estáveis tem um efeito benéfico adicional.

Palavras-chave: Segurança alimentar; Pobreza; Renda; Modelos de lógite.

\section{Food insecurity in Brazil after a crisis, its evolution from 2004 to 2017- 2018 and comparison with the variation of poverty}

The Brazilian Institute of Geography and Statistics (IBGE) collected data for the application of the Brazilian Food Insecurity Scale (EBIA) in supplements of the National Household Sample Survey (PNAD) in 2004, 2009 and 2013. With the disclosure of the data obtained in the new Family Budget Survey (POF 2017-2018), researchers now have a series of four years of data on the subject throughout the Brazilian territory. Food insecurity decreased from 2004 to 2013, but grew in 2017-2018. Data indicate that severe food insecurity in 2017-2018 is higher than in 2013, but is below the value observed in 2009. However, food insecurity of any degree in 2017-2018 exceeds even the value observed in 2004. Analyzing data by Federation Unit shows the strong association of food insecurity with income distribution and particularly with poverty measures. It turns out, with data from the PNAD, that the level of poverty fell from 2003 to 2014 and, although it rose from 2014 to 2017-2018, in these years it is substantially

1 Professor Sênior da Escola Superior de Agricultura "Luiz de Queiroz" da USP e docente aposentado do Instituto de Economia da UNICAMP. Endereço para correspondência: Caixa Postal. n. 9 (ESALQ) . CEP. 13418-900 Piracicaba, São Paulo. E-mail: hoffmannr@usp.br. ID ORCID: http://orcid.org/0000-0002-2077-8202 
below the value observed in 2004. It is argued that the great growth of light food insecurity from 2013 to 2017 2018 is largely due to the partially subjective nature of EBIA. In another section of the article, the microdata of the POF 2017-2018 are used to verify, through logit models, what are the main determinants of the probability of food insecurity in a household. The results show that increased income and schooling are basic instruments to reduce food insecurity and that more stable incomes have an additional beneficial effect.

Keywords: Food security; Poverty; Income; Logit models.

Submetido em: $14 / 12 / 2020$

Aceito em: 18/02/2021

\section{INTRODUÇÃO}

Com a divulgação dos dados da Pesquisa de Orçamentos Familiares (POF) 2017-2018 sobre segurança alimentar, dispomos, agora, de uma sequência de quatro levantamentos nacionais sobre o tema. Os levantamentos anteriores foram realizados por meio de questionários complementares na Pesquisa Nacional por Amostra de Domicílios (PNAD) em 2004, 2009 e 2013. Como explicado pormenorizadamente na publicação do IBGE[ [1], em cada domicílio da amostra são obtidas repostas a um conjunto de 14 perguntas e o domicílio é classificado em quatro categorias (com segurança alimentar ou com insegurança leve, moderada ou grave) em função do número de respostas afirmativas, levando em consideração se o domicílio tem ou não morador com menos de 18 anos de idade, de acordo com a Escala Brasileira de Insegurança Alimentar (EBIA). Para o domicílio ser classificado como tendo segurança alimentar, nenhuma das 14 perguntas pode ser respondida afirmativamente ${ }^{2}$. A primeira pergunta, por exemplo, é "Nos últimos três meses os moradores desse domicilio tiveram a preocupação de que os alimentos acabassem antes de poderem comprar ou receber mais comida?”.

A Tabela 1 mostra a evolução das porcentagens de domicílios e de pessoas nas diversas categorias de segurança alimentar com base nos quatro levantamentos nacionais disponíveis, cobrindo $\mathrm{O}$ período de 2004 a 2017-2018 3 . A porcentagem de pessoas com insegurança alimentar é sempre maior do que a respectiva porcentagem de domicilios, porque os domicílios com segurança alimentar têm, em média, menor número de moradores ${ }^{4}$. Observa-se que a proporção de domićlios com segurança alimentar cresce de 65,1\% em 2004 para 77,4\% em 2013, mas cai para 63,3\% em 2017-2018, um nível inferior ao observado em 2004. A porcentagem de moradores nos domicílios com segurança alimentar também diminui de 60,1\% em 2004 para 59,0\% em 2017-2018. O acesso dos brasileiros aos alimentos em 2017-2018 estaria pior do que em 2004?

Tabela 1. Porcentagem de domicílios particulares e de moradores conforme categorias de segurança alimentar. Brasil: 2004, 2009, 2013 e 2017-2018

\begin{tabular}{|c|c|c|c|c|c|c|c|c|}
\hline \multirow{2}{*}{$\begin{array}{c}\text { Categorias de segurança } \\
\text { alimentar }\end{array}$} & \multicolumn{4}{|c|}{ Porcentagem de domicílios } & \multicolumn{4}{|c|}{ Porcentagem de pessoas } \\
\hline & 2004 & 2009 & 2013 & $2017 / 18$ & 2004 & 2009 & 2013 & $2017 / 18$ \\
\hline Segurança alimentar & 65,1 & 69,8 & 77,4 & 63,3 & 60,1 & 65,9 & 74,2 & 59,0 \\
\hline Insegurança leve & 18,0 & 18,7 & 14,8 & 24,0 & 20,3 & 20,9 & 17,1 & 27,0 \\
\hline Insegurança moderada & 9,9 & 6,5 & 4,6 & 8,1 & 11,3 & 7,4 & 5,1 & 9,0 \\
\hline Insegurança grave & 6,9 & 5,0 & 3,2 & 4,6 & 8,2 & 5,8 & 3,6 & 5,0 \\
\hline
\end{tabular}

Fonte: IBGE, 2020[1].

\footnotetext{
${ }^{2} \mathrm{Na}$ POF as informações são coletadas em cada Unidade de Consumo (UC), que equivale a uma família. Um domicilio pode ter mais de uma UC. Para permitir a comparação com os dados anteriores sobre segurança alimentar obtidos na PNAD, na qual a unidade básica é o domicílio, o IBGE optou por atribuir ao domicílio o resultado obtido na sua UC principal (IBGE, 2020[1]) 3 Vamos admitir aqui, da mesma maneira que é feito na própria publicação do IBGE[1], que os resultados obtidos com a aplicação da EBIA na POF $2017-2018$ são comparáveis aos obtidos anteriormente na PNAD. Mas não se pode descartar a possibilidade de que o fato de as perguntas da EBIA estarem, na POF, inseridas em um conjunto muito diferente de questões (muitas questões sobre alimentação e perguntas mais detalhadas sobre rendimentos) afetem os resultados relativos à insegurança alimentar.

${ }_{4}^{4}$ Conforme a POF 2017-2018, o Brasil tinha 68,86 milhões de domicílios com 217,10 milhões de pessoas. O número médio de pessoas por domicílio era 3,01 para toda a população, 2,80 nos domicílios com segurança alimentar e nos domićlios com insegurança alimentar leve, moderada ou grave era 3,39, 3,32 e 3,28, respectivamente.
} 
Observa-se, na Tabela 1, que apenas a insegurança alimentar leve é, em 2017-2018, maior do que em 2004. No caso da insegurança alimentar grave, a porcentagem de domicílios ou de pessoas nessa condição em 2017-2018 supera o valor referente a 2013, mas fica abaixo da porcentagem observada em 2009 e substancialmente abaixo do valor correspondente em 2004. Vamos argumentar que, acompanhando o que ocorreu com a pobreza, a segurança alimentar deve ter piorado no período 2014-2017, revertendo melhoras conquistadas até 2014, mas não ao ponto de tornar o grau efetivo de insegurança alimentar em 2017-2018 pior do que o de 2004; o crescimento na insegurança alimentar geral (particularmente da insegurança leve) medido pela EBIA de 2004 a 2017-2018 se deve, em parte, ao caráter subjetivo do método de medida. Preliminarmente, na próxima seção, mostramos a forte associação entre pobreza e insegurança alimentar nas 27 Unidades da Federação.

\section{Renda e Insegurança Alimentar nas unidades da federação}

Sempre tendo por base os dados coletados na POF 2017-2018, a Tabela 2 mostra as medidas de insegurança alimentar e a Tabela 3 apresenta várias características da distribuição da Renda Domiciliar Per Capita (RDPC) nas 27 Unidades da Federação. Verificase que a RDPC média, expressa em reais de janeiro de 2018, fica abaixo de $\mathrm{R} \$ 1.500 \mathrm{em}$ todas as Unidades da Federação (UF) do Norte e do Nordeste e supera R\$ 1.700 nas UF do Sudeste, do Sul e do Centro-Oeste, destacando-se o Distrito Federal com RDPC média acima de $\mathrm{R} \$$ 4.000. O Distrito Federal também se destaca pela maior desigualdade na distribuição da RDPC, sendo a única UF cujo índice de Gini supera 0,6. As medidas de pobreza foram calculadas adotando uma linha de pobreza de $\mathrm{R} \$ 500$ (em reais de janeiro de 2018). Assim, a medida $H$ é a porcentagem de pessoas com RDPC igual ou menor do que $\mathrm{R} \$ 500$ e, sendo $m$ a RDPC média dessas pessoas, a razão de insuficiência de renda é

$$
I=\frac{500-m}{500}
$$

Como $H$ é uma medida da extensão da pobreza e $I$ é uma medida da sua intensidade (mostrando em quanto a renda média dos pobres fica abaixo da linha de pobreza), o produto $H I$ é uma medida de pobreza que leva em consideração tanto a extensão como a intensidade da pobreza. O índice de Foster, Greer e Thorbecke (FGT) é ainda mais sofisticado, levando em consideração a desigualdade da distribuição da renda entre os pobres; sendo $C$ o coeficiente de variação da RDPC entre os pobres, temos

$$
\mathrm{FGT}=H\left[I^{2}+(1-I)^{2} C^{2}\right]
$$

Observa-se, na Tabela 3, que o valor de $H I$ é igual ou maior do que 6,7 em todas as UFs do Norte e do Nordeste e fica abaixo de 6,1 nas demais UFs; os menores valores são os referentes a Santa Catarina e ao Rio Grande do Sul.

A Figura 1 ilustra a forte relação entre insegurança alimentar e pobreza que, em seguida, será avaliada por meio do cálculo de coeficientes de correlação e análise de regressão. 
Tabela 2. Insegurança alimentar nas Unidades da Federação (UF) em 2017-2018

\begin{tabular}{|c|c|c|c|c|c|c|}
\hline \multirow[b]{2}{*}{ UF } & \multirow{2}{*}{$\begin{array}{c}\text { População } \\
(1000)\end{array}$} & \multicolumn{5}{|c|}{$\%$ de pessoas em domicílios com insegurança alimentar } \\
\hline & & $\begin{array}{l}\text { De qualquer } \\
\text { grau }\end{array}$ & Leve & Moderada & $\begin{array}{c}\text { Moderada }+ \\
\text { Grave }\end{array}$ & Grave \\
\hline Rondônia & 1.738 & 37,10 & 27,63 & 6,68 & 9,46 & 2,78 \\
\hline Acre & 847 & 61,50 & 32,93 & 14,37 & 28,57 & 14,19 \\
\hline Amazonas & 3.893 & 71,02 & 34,02 & 21,03 & 37,00 & 15,97 \\
\hline Roraima & 507 & 43,00 & 25,16 & 6,81 & 17,84 & 11,04 \\
\hline Pará & 8.433 & 66,60 & 35,34 & 19,14 & 31,26 & 12,11 \\
\hline Amapá & 814 & 65,41 & 30,87 & 21,39 & 34,55 & 13,16 \\
\hline Tocantins & 1.535 & 48,87 & 32,26 & 11,84 & 16,60 & 4,76 \\
\hline Maranhão & 6.984 & 68,20 & 37,57 & 17,92 & 30,63 & 12,71 \\
\hline Piauí & 3.260 & 48,01 & 30,26 & 11,84 & 17,76 & 5,91 \\
\hline Ceará & 9.047 & 50,37 & 29,69 & 14,15 & 20,68 & 6,52 \\
\hline Rio Grande do Norte & 3467 & 58,96 & 36,08 & 14,74 & 22,88 & 8,13 \\
\hline Paraíba & 3.965 & 56,62 & 35,60 & 14,16 & 21,03 & 6,87 \\
\hline Pernambuco & 9.413 & 51,99 & 30,81 & 14,16 & 21,18 & 7,02 \\
\hline Alagoas & 3.310 & 60,77 & 38,52 & 13,74 & 22,25 & 8,51 \\
\hline Sergipe & 2.269 & 51,69 & 33,48 & 13,04 & 18,20 & 5,17 \\
\hline Bahia & 14.768 & 49,98 & 29,84 & 13,45 & 20,13 & 6,69 \\
\hline Minas Gerais & 20.973 & 34,15 & 24,72 & 6,38 & 9,43 & 3,05 \\
\hline Espírito Santo & 3.948 & 33,45 & 23,46 & 6,59 & 9,99 & 3,40 \\
\hline Rio de Janeiro & 17.111 & 35,02 & 24,32 & 7,02 & 10,71 & 3,69 \\
\hline São Paulo & 45.353 & 34,85 & 26,90 & 5,47 & 7,95 & 2,48 \\
\hline Paraná & 11.295 & 25,59 & 20,45 & 2,95 & 5,15 & 2,20 \\
\hline Santa Catarina & 7.022 & 15,39 & 10,88 & 2,27 & 4,50 & 2,23 \\
\hline Rio Grande do Sul & 11.287 & 26,11 & 19,01 & 4,54 & 7,10 & 2,57 \\
\hline Mato Grosso do Sul & 2.665 & 41,36 & 28,62 & 7,47 & 12,74 & 5,27 \\
\hline Mato Grosso & 3.369 & 34,56 & 23,70 & 7,36 & 10,86 & 3,51 \\
\hline Goiás & 6.878 & 38,99 & 26,30 & 7,58 & 12,69 & 5,11 \\
\hline Distrito Federal & 2.953 & 35,10 & 23,82 & 6,85 & 11,28 & 4,43 \\
\hline Brasil & 207.103 & 40,98 & 27,04 & 8,97 & 13,94 & 4,97 \\
\hline
\end{tabular}

Fonte: IBGE: POF (2017-2018) 
Tabela 3. Média, índice de Gini e medidas de pobreza (proporção de pobres $(H)$, razão de insuficiência de renda $(I)$, medida HI e índice de Foster, Greer e Thorbecke (FGT) para uma linha de pobreza ( $\Rightarrow$ de $\mathrm{R} \$ 500$ per capita) da distribuição da renda domiciliar per capita (RDPC) em cada Unidade da Federação (UF)

\begin{tabular}{|c|c|c|c|c|c|c|}
\hline \multirow{2}{*}{ UF } & \multirow{2}{*}{$\begin{array}{l}\text { RDPC média } \\
(\mathrm{R} \$)^{(1)}\end{array}$} & \multirow{2}{*}{$\begin{array}{l}\text { Índice } \\
\text { de Gini }\end{array}$} & \multicolumn{4}{|c|}{ Medidas de pobreza para $z=500$} \\
\hline & & & $H$ & $I$ & $H I$ & FGT \\
\hline Rondônia & 1.264 & 0,442 & 20,80 & 0,370 & 7,69 & 4,05 \\
\hline Acre & 1.143 & 0,539 & 39,67 & 0,346 & 13,71 & 6,88 \\
\hline Amazonas & 1.014 & 0,555 & 43,88 & 0,442 & 19,40 & 11,24 \\
\hline Roraima & 1.298 & 0,554 & 36,22 & 0,387 & 14,01 & 7,66 \\
\hline Pará & 949 & 0,515 & 42,59 & 0,431 & 18,35 & 10,58 \\
\hline Amapá & 1.207 & 0,506 & 33,29 & 0,322 & 10,71 & 4,90 \\
\hline Tocantins & 1.005 & 0,484 & 34,16 & 0,401 & 13,71 & 7,48 \\
\hline Maranhão & 784 & 0,463 & 44,91 & 0,417 & 18,73 & 10,49 \\
\hline Piauí & 1.056 & 0,468 & 32,48 & 0,330 & 10,73 & 5,00 \\
\hline Ceará & 1.048 & 0,505 & 36,67 & 0,398 & 14,60 & 7,89 \\
\hline Rio Grande do Norte & 1.267 & 0,489 & 28,60 & 0,323 & 9,23 & 4,27 \\
\hline Paraíba & 1.105 & 0,534 & 38,95 & 0,402 & 15,65 & 8,52 \\
\hline Pernambuco & 1.234 & 0,519 & 32,13 & 0,373 & 11,97 & 6,30 \\
\hline Alagoas & 860 & 0,508 & 47,75 & 0,407 & 19,41 & 10,81 \\
\hline Sergipe & 1.462 & 0,499 & 23,23 & 0,289 & 6,70 & 2,90 \\
\hline Bahia & 1.262 & 0,522 & 31,46 & 0,355 & 11,17 & 5,49 \\
\hline Minas Gerais & 1.736 & 0,473 & 13,46 & 0,292 & 3,93 & 1,70 \\
\hline Espírito Santo & 1.791 & 0,508 & 16,86 & 0,330 & 5,57 & 2,66 \\
\hline Rio de Janeiro & 1.939 & 0,527 & 17,10 & 0,354 & 6,05 & 3,05 \\
\hline São Paulo & 2.556 & 0,529 & 9,22 & 0,305 & 2,81 & 1,28 \\
\hline Paraná & 1.996 & 0,486 & 12,89 & 0,371 & 4,78 & 2,65 \\
\hline Santa Catarina & 2.136 & 0,424 & 6,91 & 0,308 & 2,13 & 0,95 \\
\hline Rio Grande do Sul & 2.329 & 0,468 & 8,37 & 0,233 & 1,95 & 0,72 \\
\hline Mato Grosso do Sul & 1.897 & 0,459 & 11,10 & 0,266 & 2,96 & 1,19 \\
\hline Mato Grosso & 1.815 & 0,487 & 15,11 & 0,292 & 4,41 & 2,00 \\
\hline Goiás & 1.881 & 0,489 & 12,50 & 0,269 & 3,36 & 1,42 \\
\hline Distrito Federal & 4.116 & 0,601 & 8,83 & 0,357 & 3,16 & 1,72 \\
\hline Brasil & 1.808 & 0,535 & 20,38 & 0,368 & 7,50 & 3,90 \\
\hline
\end{tabular}

Fonte: IBGE: POF (2017-2018)

(1) Em reais de janeiro de 2018. 
Figura 1. Porcentagem de pessoas com insegurança alimentar de qualquer grau e proporção de pobres para linha de pobreza de R \$ 500 per capita nas 27 Unidades da Federação em 2017-2018

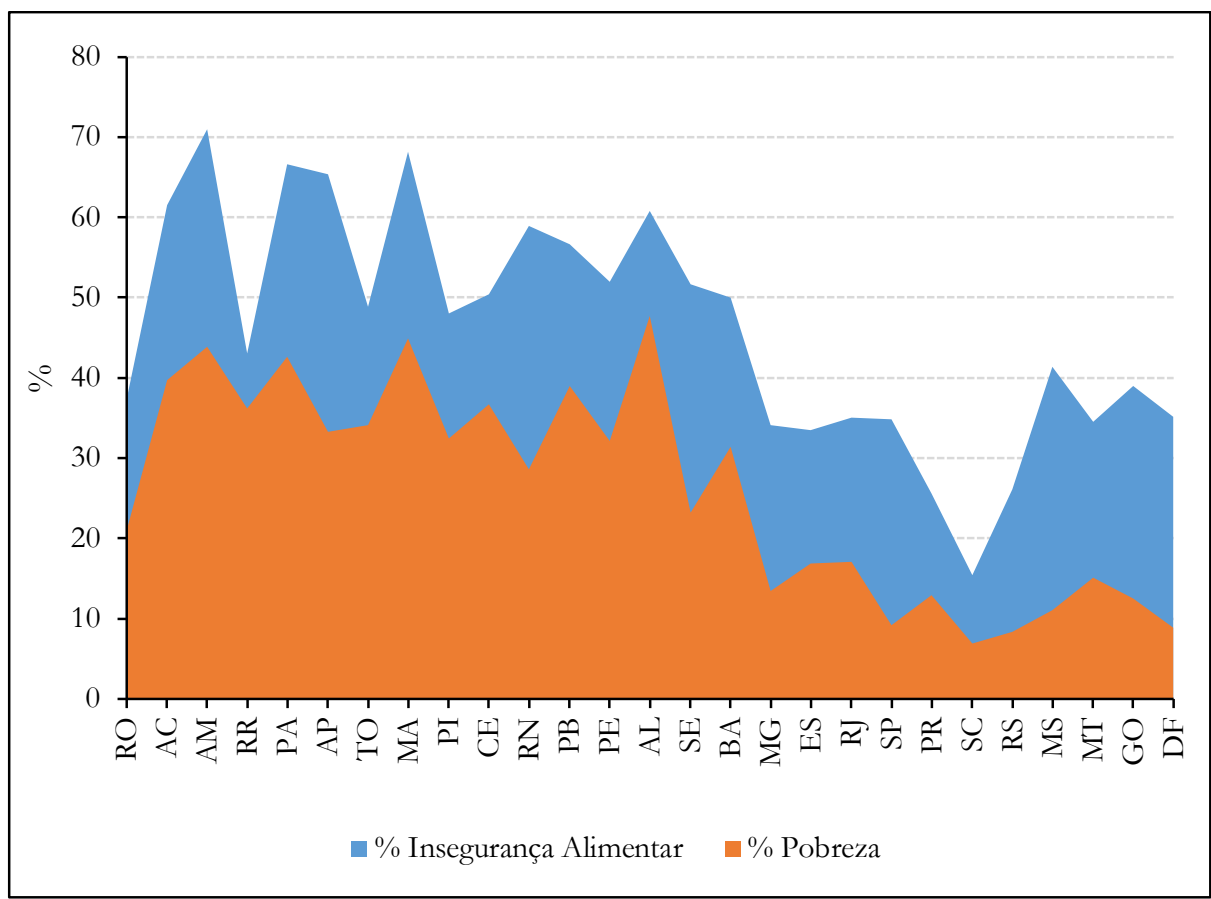

Fonte: Elaborada a partir de microdados da POF 2017-2018.

Para facilitar a exposição, vamos definir as seguintes variáveis, considerando seus valores nas 27 Unidades da Federação:

$I_{I A}=$ porcentagem de pessoas com insegurança alimentar de qualquer grau;

$I_{M G}=$ porcentagem de pessoas com insegurança alimentar moderada ou grave;

$I_{G R}=$ porcentagem de pessoas com insegurança alimentar grave;

$y=$ logaritmo neperiano da RDPC média da UF;

$G$ = índice de Gini da distribuição da RDPC;

$H=$ proporção de pobres para uma linha de pobreza de $\mathrm{R} \$ 500$ per capita;
$H I=$ produto da proporção de pobres pela razão de insuficiência de renda.

Em trabalhos anteriores com os dados das PNADs de 2004, 2009 e 2013, já ressaltamos a estreita relação da insegurança alimentar com a RDPC (Hoffmann $\left.{ }^{2,3,4]}\right)$. Isso é confirmado pela análise dos dados por UF apresentados nas Tabelas 2 e 3 . As correlações de $y$ com $I_{I A}, \quad I_{M G}$ e $I_{G R}$ são, respectivamente, $-0,831,-0,864$ e $-0,821$, todas fortemente significativas. Vamos denominar uma estimativa de "fortemente significativa" quando a probabilidade caudal ("valor $p$ ") associado à respectiva hipótese de nulidade for inferior a $0,01 \%$. Todas as análises estatísticas nesta seção são elaboradas ponderando os dados de cada UF pela respectiva população, respeitando as diferenças na sua importância relativa.

Fixada a renda média, a pobreza tende a crescer com a desigualdade e isso deve causar maior insegurança alimentar. Realmente, verifica-se que as 
correlações do índice de Gini $(G)$ com $I_{I A}, I_{M G}$ e $I_{G R}$ são positivas. Mas essas correlações não são estatisticamente significativas ao nível de $10 \%$. Entretanto, fazendo uma regressão linear múltipla de $I_{A}$ contra $y$ e $G$, os coeficientes de regressão estimados $(-31,9$ e 177,7$)$ são ambos fortemente significativos. Isso sugere examinar a correlação das medidas de insegurança alimentar com as medidas de pobreza. Verifica-se que todas as correlações entre, de um lado, as medidas de insegurança alimentar $I_{I A}, I_{M G}$ e $I_{G R}$ e, de outro lado, as medidas de pobreza $H, H I$ e o índice FGT são positivas e fortemente significativas; quase todas são maiores do que 0,9 ; as correlações entre $I_{M G}$ e $H$ ou $H I$ superam 0,93 .

Uma vez constatada a forte associação entre insegurança alimentar e pobreza, na próxima seção examinamos a evolução dessas variáveis no Brasil ao longo dos anos.

\section{Variação da renda, da pobreza e da insegurança alimentar}

As Figuras 2 e 3 mostram a evolução da medida de pobreza FGT no Brasil de 1995 a 2019, usando dados da PNAD. O objetivo é mostrar as mudanças nessa característica básica da distribuição da RDPC para um período bastante longo. A série se inicia em 1995 para evitar dados anteriores muito afetados por uma inflação descontrolada. A PNAD tradicional foi realizada até 2015, mas até 2003 não cobria a área rural da antiga região Norte (Rondônia, Acre, Amazonas, Roraima, Pará e Amapá). A PNAD tradicional, no período 1995-2019 teve sempre como referência o mês de setembro. A partir de 2012 o IBGE passou a fazer a PNAD Contínua, aperfeiçoando o processo de amostragem e com coleta de dados distribuída ao longo do ano. Optamos, então, por considerar três séries:

(a) Dados da PNAD tradicional de 1995 a 2015, excluindo sempre a área rural da antiga região Norte; (b) Dados da PNAD tradicional de 2004 a 2015 abrangendo todo o País;

(c) Dados anuais da PNAD Contínua de 2012 a 2019 abrangendo todo o País.

Observa-se, nas Figuras 2 e 3, que a inclusão da área rural da antiga região Norte eleva a medida de pobreza, pois se trata de uma população comparativamente mais pobre; mas o efeito é muito pequeno e não afeta em nada o comportamento da evolução da pobreza ao longo dos anos. Já as mudanças metodológicas da PNAD tradicional para a PNAD Contínua têm efeitos maiores, mas observa-se grande coerência na evolução das séries (b) e (c).

As Figuras 2 e 3 ilustram, também, a evolução, de 2004 a 2017-2018, das porcentagens de pessoas com três níveis de insegurança alimentar: geral $\left(I_{I A}\right)$, moderada ou grave $\left(I_{M G}\right)$ e considerando apenas a insegurança alimentar grave $\left(I_{G R}\right)$. Note-se que as medidas de insegurança alimentar obtidas da POF 2017-2018 são atribuídas ao ano de 2018.

Observa-se, nessas figuras, substancial redução da pobreza de 2003 a 2014, forte crescimento de 2014 a 2017 e relativa estabilidade de 2017 a 2019. Interessa-nos, particularmente, o nível de pobreza em 2004, 2009, 2013 e 2018, anos para os quais dispomos das medidas de insegurança alimentar. A pobreza diminui de 2004 a 2009 e também de 2009 a 2013, o que é perfeitamente coerente com a redução da proporção de pessoas com algum grau de insegurança alimentar de 39,9\% em 2004 para 34,1\% em 2009 e 25,8\% em 2013. O nível de pobreza em 2018 é substancialmente maior do que em 2013, o que, novamente, é coerente com o expressivo aumento da insegurança alimentar no período. Entretanto, embora a proporção de domicílios ou de pessoas com algum grau de insegurança alimentar em 2017-2018 seja maior do que em 2004, as Figuras 2 e 3 mostram queda na pobreza nesse período. 
Figura 2. Evolução do índice de pobreza FGT da distribuição da renda domiciliar per capita no Brasil, de 1995 a 2019, adotando uma linha de pobreza de $\mathrm{R} \$ 400$ (em reais do quarto trimestre de 2019), e evolução das porcentagens de pessoas com insegurança alimentar de qualquer grau $\left(I_{I A}\right)$, Moderada + Grave $\left(I_{M G}\right)$ ou Grave $\left(I_{G R}\right)$, no Brasil, de 2004 a 2018

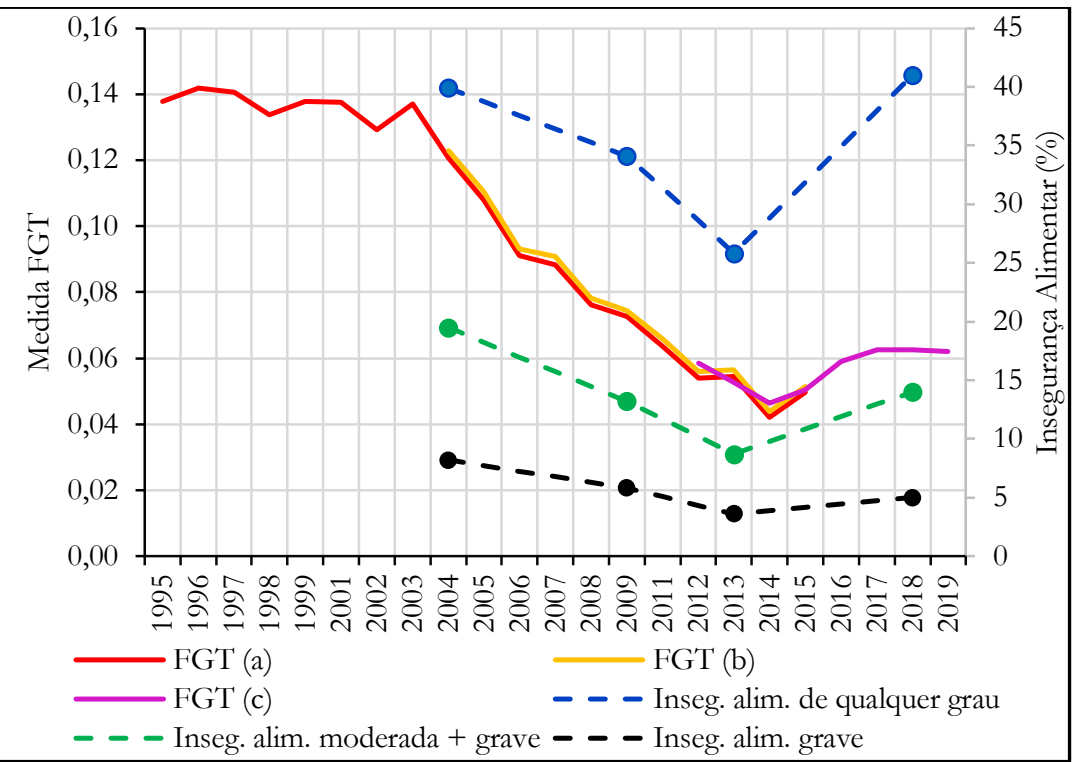

Fonte: Hoffmann, 2020[5,6], incluindo resultados para insegurança alimentar.

Figura 3. Evolução do índice de pobreza FGT da distribuição da renda domiciliar per capita no Brasil, de 1995 a 2019, adotando uma linha de pobreza de $\mathrm{R} \$ 200$ (em reais do quarto trimestre de 2019), e evolução da porcentagem de pessoas com insegurança alimentar de qualquer grau $\left(I_{I A}\right)$, Moderada + Grave $\left(I_{M G}\right)$ ou Grave $\left(I_{G R}\right)$, no Brasil, de 2004 a 2018

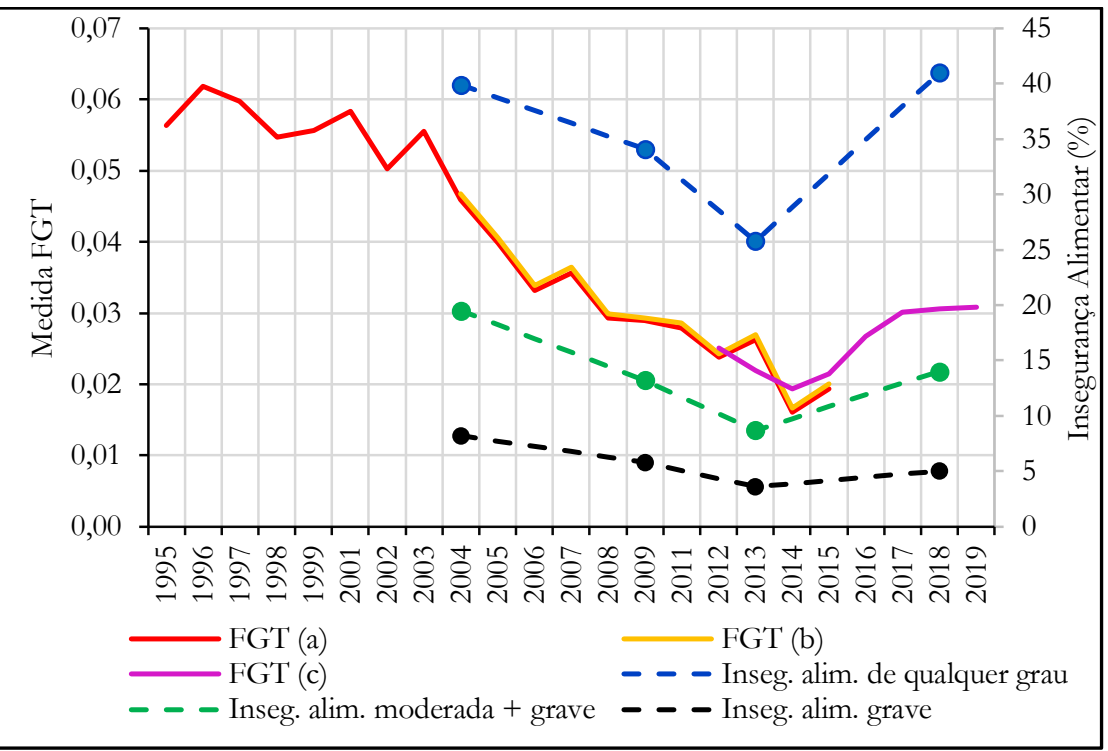

Fonte: Hoffmann, 2020[5,6], incluindo resultados para insegurança alimentar. 
Na Figura 2, considerando a linha de pobreza de $\mathrm{R} \$ 400$, o valor do índice FGT em 2017-2018 é semelhante ao observado em 2011; na Figura 3, adotando uma linha de pobreza de $\mathrm{R} \$ 200$, o índice FGT em 2017-2018 é similar ao observado em 2009. Verifica-se, portanto, que ao considerar a série de quatro valores da insegurança alimentar geral, o comportamento ao longo dos anos é distinto do comportamento do nível de pobreza. Por outro lado, a série de quatro valores para a insegurança alimentar grave se mostra bem mais coerente com a variação da pobreza, com valor em $2017-2018$ (5,0\% das pessoas) inferior ao observado em 2004 ( $8,2 \%$ das pessoas) e semelhante ao de 2009 (5,8\% das pessoas). Tudo indica que o forte crescimento da insegurança alimentar de qualquer grau de 2013 a 2017-2018 se deve, em parte, ao caráter parcialmente subjetivo da medida de insegurança alimentar, particularmente da insegurança alimentar leve e, com menos intensidade, da moderada. Depois de um período com substancial redução da pobreza (2003-2014), muitas famílias se habituaram a dispender mais com alimentação e a óbvia insatisfação com a piora da situação econômica a partir de 2014 faz com que sejam mais facilmente classificados em situação de insegurança alimentar ao responder as 14 perguntas da EBIA.

A comparação entre o consumo alimentar pessoal na POF 2008-2009 e na POF 2017-2018, apresentada em publicação do IBGE[7] (p. 68-104), mostra mudanças relevantes no período, como, por exemplo, a redução da prevalência da alimentação fora do domicílio e redução da sua contribuição para o consumo energético total, mas não indica que tenha ocorrido uma piora geral na qualidade da alimentação. Esses dados não contradizem, portanto, a afirmativa de que o aumento da insegurança alimentar leve e moderada de 2009 a 2017-2018 observada na Tabela 1 está associada ao caráter parcialmente subjetivo da EBIA.

\section{A Insegurança Alimentar em 11 estratos de renda domiciliar per capita}

Para verificar como a insegurança alimentar varia com o nível de renda, vamos dividir a população (de domicílios e de pessoas) em 11 estratos de RDPC, como mostra a Tabela 4. Trata-se de pequena modificação na divisão em 10 estratos adotada em Hoffmann e Vaz ${ }^{[8]}$, onde o limite superior do primeiro estrato é igual a $\mathrm{R} \$ 400$; aqui esse estrato foi subdividido em dois, dado o interesse de examinar o que ocorre com a insegurança alimentar entre os muito pobres. 
Tabela 4. Número e porcentagem de domicílios e de pessoas, número médio de pessoas por domicílio, porcentagem da renda total e RDPC média em 11 estratos de RDPC, Brasil, 2017-2018

\begin{tabular}{|c|c|c|c|c|c|c|c|}
\hline \multirow[b]{2}{*}{ Estrato de RDPC } & \multicolumn{2}{|c|}{ Domicílios } & \multicolumn{2}{|c|}{ Pessoas } & \multirow{2}{*}{$\begin{array}{c}\text { Pessoa por } \\
\text { domicílio }\end{array}$} & \multirow{2}{*}{$\begin{array}{c}\% \text { da renda } \\
\text { total }\end{array}$} & \multirow{2}{*}{$\begin{array}{l}\text { RDPC } \\
\text { média (1) }\end{array}$} \\
\hline & $\begin{array}{c}\mathrm{n}^{\mathrm{o}} \\
(1000)\end{array}$ & $\%$ & $\begin{array}{c}\mathrm{n}^{\mathrm{o}} \\
(1000)\end{array}$ & $\%$ & & & \\
\hline De 0 a 200 & 1.818 & 2,64 & 8.126 & 3,92 & 4,47 & 0,29 & 135,23 \\
\hline Mais de 200 a 400 & 5.111 & 7,42 & 21.537 & 10,40 & 4,21 & 1,75 & 304,09 \\
\hline Mais de 400 a 700 & 9.521 & 13,83 & 35.615 & 17,20 & 3,74 & 5,20 & 546,70 \\
\hline Mais de 700 a 1000 & 9.402 & 13,65 & 31.617 & 15,27 & 3,36 & 7,11 & 842,42 \\
\hline Mais de 1000 a 1300 & 8.755 & 12,71 & 25.453 & 12,29 & 2,91 & 7,79 & $1.145,66$ \\
\hline Mais de 1300 a 1600 & 7.043 & 10,23 & 18.768 & 9,06 & 2,66 & 7,23 & $1.442,37$ \\
\hline Mais de 1600 a 2000 & 6.563 & 9,53 & 17.012 & 8,21 & 2,59 & 8,11 & $1.784,86$ \\
\hline Mais de 2000 a 3000 & 8.704 & 12,64 & 21.322 & 10,30 & 2,45 & 13,77 & $2.418,62$ \\
\hline Mais de 3000 a 5000 & 6.301 & 9,15 & 15.278 & 7,38 & 2,42 & 15,41 & $3.778,28$ \\
\hline Mais de 5000 a 10000 & 3.852 & 5,59 & 8.692 & 4,20 & 2,26 & 15,94 & $6.869,93$ \\
\hline Mais de 10000 & 1.791 & 2,60 & 3.683 & 1,78 & 2,06 & 17,40 & $17.696,66$ \\
\hline Brasil (total) & 68.862 & 100,00 & 207.104 & 100,00 & 3,01 & 100,00 & $1.808,46$ \\
\hline
\end{tabular}

Fonte: IBGE: POF (2017-2018)

(1) Em reais de janeiro de 2018.

Observa-se, na Tabela 4, que o número de pessoas por domicilio cai sistematicamente à medida que a RDPC aumenta.

Na POF 2017-2018 questionou-se, em cada Unidade de Consumo, como era avaliado o padrão de vida em relação a alimentação, com três respostas possíveis: "Bom", "Satisfatório" ou "Ruim". Seguindo o mesmo procedimento utilizado para a classificação conforme a EBIA, atribui-se ao domicílio o resultado obtido na sua UC principal. Há perguntas semelhantes para avaliação das condições de vida referentes a moradia, vestuário, educação, saúde e lazer.

A Tabela 5 mostra, para cada um dos 11 estratos de RDPC, a proporção de domicílios nas quatro categorias de segurança alimentar e conforme as três possíveis respostas à pergunta sobre o padrão de vida na alimentação. A Tabela 6 mostra as porcentagens correspondentes considerando o número de pessoas. De acordo com o esperado, verifica-se que, à medida que aumenta a RDPC, ocorre: a) Crescimento sistemático das porcentagens com segurança alimentar e das porcentagens com bom padrão de vida na alimentação;

b) Diminuição sistemática das porcentagens com insegurança moderada ou grave;

c) Diminuição, em geral, das porcentagens com insegurança alimentar leve e das porcentagens com padrão de vida na alimentação avaliado como "satisfatório" ou "ruim".

Curiosamente, há aumento da proporção com insegurança alimentar quando se passa do primeiro para o segundo estrato. Fenômeno semelhante foi observado em análise dos dados da PNAD de 2013 e atribuído ao caráter parcialmente subjetivo da EBIA (Hoffmann, 2014[4], p. 427). 
Tabela 5. Número de domicílios e sua distribuição percentual em quatro categorias de segurança alimentar e em três categorias de avaliação do padrão de vida em relação a alimentação conforme estratos de RDPC, Brasil, 2017-2018

\begin{tabular}{|c|c|c|c|c|c|c|c|c|}
\hline \multirow{2}{*}{ Estrato de RDPC } & \multirow{2}{*}{$\begin{array}{l}\text { Domicílios } \\
\text { no } 0 \text { (1000) }\end{array}$} & \multirow{2}{*}{$\begin{array}{c}\% \text { com } \\
\text { segurança } \\
\text { alimentar }\end{array}$} & \multicolumn{3}{|c|}{$\%$ com inseg. alimentar } & \multicolumn{3}{|c|}{$\begin{array}{l}\text { \% com padrão de vida } \\
\text { (alimentação) }\end{array}$} \\
\hline & & & leve & moder. & grave & bom & satisf. & ruim \\
\hline De 0 a 200 & 1.818 & 26,7 & 32,3 & 21,1 & 19,9 & 39,3 & 45,6 & 15,2 \\
\hline Mais de 200 a 400 & 5.111 & 29,1 & 37,8 & 20,3 & 12,8 & 41,7 & 46,5 & 11,8 \\
\hline Mais de 400 a 700 & 9.521 & 42,7 & 35,8 & 14,4 & 7,1 & 49,6 & 41,8 & 8,7 \\
\hline Mais de 700 a 1000 & 9.402 & 52,8 & 32,1 & 9,8 & 5,3 & 54,1 & 39,1 & 6,8 \\
\hline Mais de 1000 a 1300 & 8.755 & 61,3 & 27,5 & 7,2 & 4,0 & 56,6 & 38,3 & 5,2 \\
\hline Mais de 1300 a 1600 & 7.043 & 68,1 & 22,5 & 6,2 & 3,2 & 59,1 & 36,2 & 4,6 \\
\hline Mais de 1600 a 2000 & 6.563 & 72,2 & 20,4 & 5,0 & 2,3 & 63,5 & 32,9 & 3,6 \\
\hline Mais de 2000 a 3000 & 8.704 & 79,0 & 15,9 & 3,6 & 1,5 & 69,3 & 27,8 & 2,9 \\
\hline Mais de 3000 a 5000 & 6.301 & 86,9 & 9,8 & 2,1 & 1,2 & 74,3 & 24,2 & 1,5 \\
\hline Mais de 5000 a 10000 & 3.852 & 92,8 & 5,9 & 0,8 & 0,5 & 76,7 & 20,9 & 2,4 \\
\hline Mais de 10000 & 1.791 & 97,5 & 2,1 & 0,4 & 0,0 & 85,4 & 13,9 & 0,7 \\
\hline Brasil (total) & 68.862 & 63,3 & 24,0 & 8,1 & 4,6 & 59,7 & 34,7 & 5,6 \\
\hline
\end{tabular}

Fonte: IBGE: POF (2017-2018)

Tabela 6. Número de pessoas e sua distribuição percentual em quatro categorias de segurança alimentar e em três categorias de avaliação do padrão de vida em relação a alimentação conforme estratos de RDPC, Brasil, 2017-2018

\begin{tabular}{|c|c|c|c|c|c|c|c|c|}
\hline \multirow[t]{2}{*}{ Estrato de RDPC } & \multirow{2}{*}{$\begin{array}{l}\text { Pessoas } \\
\text { oㅡ (1000) }\end{array}$} & \multirow{2}{*}{$\begin{array}{l}\text { \% com } \\
\text { segurança } \\
\text { alimentar }\end{array}$} & \multicolumn{3}{|c|}{$\%$ com inseg. alimentar } & \multicolumn{3}{|c|}{$\begin{array}{l}\text { \% com padrão de vida } \\
\text { (alimentação) }\end{array}$} \\
\hline & & & leve & moder. & grave & bom & satisf. & ruim \\
\hline De 0 a 200 & 8.126 & 24,1 & 32,2 & 22,0 & 21,7 & 38,5 & 45,1 & 16,3 \\
\hline Mais de 200 a 400 & 21.537 & 26,9 & 38,9 & 21,0 & 13,1 & 41,0 & 46,8 & 12,2 \\
\hline Mais de 400 a 700 & 35.615 & 42,2 & 36,6 & 14,4 & 6,8 & 49,4 & 42,3 & 8,3 \\
\hline Mais de 700 a 1000 & 31.617 & 52,7 & 34,0 & 8,8 & 4,6 & 55,3 & 38,7 & 6,0 \\
\hline Mais de 1000 a 1300 & 25.453 & 61,0 & 29,7 & 6,4 & 2,9 & 58,2 & 37,5 & 4,3 \\
\hline Mais de 1300 a 1600 & 18.768 & 68,1 & 23,9 & 5,6 & 2,4 & 60,4 & 35,9 & 3,6 \\
\hline Mais de 1600 a 2000 & 17.012 & 73,8 & 20,5 & 4,1 & 1,6 & 65,2 & 31,9 & 2,9 \\
\hline Mais de 2000 a 3000 & 21.322 & 79,4 & 16,6 & 3,0 & 1,1 & 70,3 & 27,6 & 2,1 \\
\hline Mais de 3000 a 5000 & 15.278 & 87,5 & 10,1 & 1,7 & 0,7 & 75,6 & 23,2 & 1,1 \\
\hline Mais de 5000 a 10000 & 8.692 & 92,6 & 6,3 & 0,6 & 0,4 & 78,4 & 19,8 & 1,8 \\
\hline Mais de 10000 & 3.683 & 97,5 & 2,2 & 0,3 & 0,0 & 86,9 & 12,5 & 0,6 \\
\hline Brasil (total) & 207.104 & 59,0 & 27,0 & 9,0 & 5,0 & 58,3 & 35,9 & 5,8 \\
\hline
\end{tabular}

Fonte: IBGE: POF (2017-2018) 
Mesmo entre aqueles com RDPC acima de R\$ 10.000 há domicílios e pessoas que foram classificados como estando em situação de insegurança alimentar grave; a proporção é igual a $0,03 \%$ que, com apenas uma decimal, aparece arredondada para 0,0 nas Tabelas 5 e 6. Já no estrato de RDPC de mais de $\mathrm{R} \$ 5.000$ a R $\$$ 10.000 há $0,49 \%$ de domicílios e $0,44 \%$ de pessoas classificadas como apresentando insegurança alimentar grave. As mesmas Tabelas mostram que nesses dois estratos, que incluem os $6 \%$ da população com maiores rendas per capita, é mais substancial a porcentagem com padrão de vida da alimentação considerado "ruim", indicando que essa classificação tem um grau de subjetividade muito maior do que o da EBIA.
A Tabela 7 mostra o cruzamento das classificações conforme quatro categorias de segurança alimentar e conforme três níveis de padrão de vida na alimentação. Há clara associação entre as duas classificações; verifica-se, por exemplo, que a porcentagem de padrão "ruim" aumenta sistematicamente com o grau de insegurança alimentar. Mas é estranho que nada menos que 20,3\% dos domicílios com insegurança alimentar grave sejam classificados com padrão de vida alimentar "bom".

Tabela 7. Número e porcentagem de domicílios com padrão de vida na alimentação "Bom", "Satisfatório" ou "Ruim" em cada uma das quatro categorias de segurança alimentar, Brasil, 2017-2018

\begin{tabular}{l|r|r|r|r|r|r}
\hline \multirow{2}{*}{$\begin{array}{c}\text { Categoria de Segurança } \\
\text { Alimentar }\end{array}$} & \multicolumn{2}{|c|}{ Número de domićlios (1000) } & \multicolumn{3}{c}{ Porcentagem na linha } \\
\cline { 2 - 7 } & Bom & Satisfatório & Ruim & Bom & Satisfatório & \multirow{2}{*}{ Ruim } \\
\hline Com Segurança Alimentar & 31.221 & 11.763 & 603 & 71,6 & 27,0 & 1,4 \\
Insegurança Leve & 7.594 & 7.857 & 1.091 & 45,9 & 47,5 & 6,6 \\
Insegurança Moderada & 1.677 & 2.861 & 1.060 & 30,0 & 51,1 & 18,9 \\
Insegurança Grave & 638 & 1.436 & 1.062 & 20,3 & 45,8 & 33,9 \\
\hline Total & 41.130 & 23.918 & 3.815 & 59,7 & 34,7 & 5,6 \\
\hline
\end{tabular}

Fonte: IBGE: POF (2017-2018)

\section{Outros determinantes da Insegurança Alimentar}

Seja $P_{i}$ a probabilidade de o $i$-ésimo domicílio apresentar certo tipo de insegurança alimentar. Por definição, o lógite correspondente é igual a

$$
Y_{i}=\ln \frac{P_{i}}{1-P_{i}}
$$

Note-se que o lógite é uma transformação monotonicamente crescente da probabilidade $P_{i}$. No modelo de lógite admite-se que $Y_{i}$ é uma função linear das diversas variáveis $\left(X_{h i}, \operatorname{com} h=1,2, \ldots, k\right)$ que afetam a probabilidade $P_{i}$.

$$
Y_{i}=\ln \frac{P_{i}}{1-P_{i}}=\alpha+\beta_{1} X_{1 i}+\beta_{2} X_{2 i}+\cdots+\beta_{k} X_{k i}+\varepsilon_{i}
$$

Assim, modelos de lógite podem ser usados para analisar como diversas variáveis afetam a probabilidade de um domicílio apresentar determinado tipo de insegurança alimentar. A qualidade do ajuste pode ser avaliada por meio do coeficiente de concordância $(c)$, associado à proporção de pares concordantes no total de pares de observações com desfechos distintos para a variável dependente.

As estimativas são obtidas a partir dos microdados da POF 2017-2018, pelo método da máxima verossimilhança, usando os fatores de ponderação fornecidos pelo IBGE e respeitando a estrutura complexa ${ }^{[9]}$ da amostra de 57.920 domicílios. 
Como variável a ser "explicada" vamos considerar a probabilidade de ocorrer qualquer grau de insegurança alimentar $\left(P_{I A}\right)$, insegurança alimentar moderada ou grave $\left(P_{M G}\right)$ ou apenas insegurança alimentar grave $\left(P_{G R}\right)$; os respectivos lógites são representados por $Y_{I A}, Y_{M G}$ e $Y_{G R}$.

Consideremos, inicialmente, um modelo de lógite com uma única variável explanatória: uma variável binária que indica se o domicílio está em área rural $\left(X_{R}=1\right)$ ou em área urbana $\left(X_{R}=0\right)$. Para qualquer grau de insegurança alimentar e com o acento circunflexo indicando valor estimado, a equação ajustada é

$$
\widehat{Y}_{I A}=-0,6126+0,4699 X_{R}, \operatorname{com} c=0,513
$$

e com os dois coeficientes fortemente significativos. $\mathrm{O}$ coeficiente de regressão positivo e significativo revela que a insegurança alimentar tende a ser maior na área rural do que na área urbana, confirmando o que mostram a Tabela 3 e o Gráfico 1 na publicação do $\operatorname{IBGE}^{[1]}$ (p. 30-31). Cabe ressaltar que, com $c$ próximo de 0,5 , a equação ajustada é praticamente inútil para previsão pois por puro acaso espera-se que metade dos pares sejam concordantes, o que corresponde a $c=$ 0,5 .

A renda média é substancialmente mais baixa nas áreas rurais do que nas áreas urbanas e já conhecemos a forte correlação negativa da insegurança alimentar com a renda. É importante saber se a maior insegurança alimentar na área rural se deve apenas à renda mais baixa ou se há algum outro efeito. $\mathrm{O}$ interessante é que quando se controla apropriadamente o efeito da RDPC, o efeito de ser rural sobre a insegurança alimentar se torna negativo, isto é, fixada a renda, o fato de um domicílio estar na área rural reduz a probabilidade de ele apresentar insegurança alimentar. Incluindo o logaritmo neperiano da RDPC do domicílio como variável explanatória ${ }^{5}$, a equação estimada fica

$$
\hat{Y}_{I A}=6,8807-1,0485 \cdot \log (\mathrm{RDPC})-0,1013 X_{R} \operatorname{com} c=0,732
$$

${ }^{5}$ Como há na amostra um domicílio com renda nula e não se define o logaritmo de zero, o ajuste da equação é feito com 57.919 observações. O caráter fortemente assimétrico da distribuição da RDPC faz com que o seu logaritmo capte melhor o efeito dessa variável sobre a insegurança alimentar.
Os dois primeiros coeficientes são fortemente significativos e a probabilidade caudal para a hipótese de nulidade do efeito de $X_{R}$ é igual a $0,1 \%$.

Em seguida vamos analisar o efeito da localização regional sobre a insegurança alimentar. As diferenças entre as cinco grandes regiões do País no que se refere à incidência dos vários graus de insegurança alimentar estão claramente expostas na publicação do $\operatorname{IBGE}^{[1]}$ (p. 32-34). Aqui vamos distinguir sete regiões, destacando o estado de São Paulo do Sudeste e o Distrito Federal do Centro-Oeste. Para analisar o efeito da localização regional por meio de um modelo de lógite, definimos seis variáveis binárias, adotando o Nordeste como base. Para distinguir os domicilios da região Norte definimos $R_{N O}=1$ para domicílio nessa região e $R_{N O}=0$ nas demais. Analogamente, definimos as variáveis binárias $R_{S E}, R_{S P}, R_{S U}, R_{C O}$ e $R_{D F}$ para distinguir as regiões Sudeste exclusive São Paulo, estado de São Paulo, Sul, Centro-Oeste exclusive Distrito Federal e Distrito Federal, respectivamente.

A Tabela 8 mostra as equações estimadas para insegurança alimentar de qualquer grau considerando apenas as seis variáveis binárias para distinguir as sete regiões ou incluindo, também, o logaritmo da RDPC como variável explanatória. Na primeira equação dessa tabela todos os seis coeficientes de regressão são fortemente significativos mostrando que, em comparação com o Nordeste, a probabilidade de insegurança alimentar é maior no Norte e menor nas demais regiões, especialmente no Sul. Com a inclusão do logaritmo da RDPC como variável explanatória, a qualidade do ajustamento melhora (o coeficiente $c$ aumenta de 0,640 para 0,745) e o valor absoluto dos efeitos regionais se reduz, mostrando que alguns eram, em grande parte, devidos às diferenças de nível de RDPC entre o Nordeste e as outras regiões analisadas ${ }^{6}$. No caso do Distrito Federal ocorre inversão do sinal da estimativa do parâmetro e ele deixa de ser estatisticamente diferente de zero, isto é, após controlar o efeito da RDPC, não há diferença estatisticamente significativa no nível de insegurança alimentar entre o Distrito Federal e o Nordeste.
${ }^{6} \mathrm{O}$ valor médio da RDPC utilizada neste artigo é R\$1.808 no Brasil, R $\$ 1.030$ no Norte, R\$ 1.126 no Nordeste, R\$ 1.824 no Sudeste exclusive SP, R\$ 2.556 no estado de São Paulo, R\$2.156 no Sul, R\$ 1.867 no Centro-Oeste exclusive DF e R \$ 4.116 no Distrito Federal. 
Tabela 8. Modelos de lógite para a probabilidade de um domicílio ter insegurança alimentar de qualquer grau, considerando sete regiões e incluindo ou não a RDPC, Brasil, 2017-2018

\begin{tabular}{l|cc|c|c|c|c}
\hline \multirow{2}{*}{ Variável } & \multicolumn{3}{c|}{ Sem RDPC } & \multicolumn{3}{c}{ Com RDPC } \\
\cline { 2 - 7 } & $\begin{array}{c}\text { Estimativa do } \\
\text { parâmetro }\end{array}$ & $\begin{array}{c}\text { Teste } \\
t\end{array}$ & $\begin{array}{c}\text { Valor- } p^{1} \\
(\%)\end{array}$ & $\begin{array}{c}\text { Estimativa do } \\
\text { parâmetro }\end{array}$ & $\begin{array}{c}\text { Teste } \\
t\end{array}$ & $\begin{array}{c}\text { Valor } p^{(1)} \\
(\%)\end{array}$ \\
\hline Constante & 0,0135 & 0,58 & 56,5 & 6,5412 & 43,67 & $*$ \\
$\log$ (RDPC) & - & - & - & $-0,9682$ & $-44,62$ & $*$ \\
Norte & 0,2665 & 5,51 & $*$ & 0,2254 & 4,32 & $*$ \\
Sudeste excl. SP & $-0,7884$ & $-16,49$ & $*$ & $-0,4224$ & $-8,93$ & $*$ \\
São Paulo & $-0,8207$ & $-14,27$ & $*$ & $-0,1947$ & $-3,50$ & $*$ \\
Sul & $-1,3592$ & $-27,98$ & $*$ & $-0,8574$ & $-15,12$ & $*$ \\
C. Oeste excl. DF & $-0,6001$ & $-9,87$ & $*$ & $-0,1696$ & $-3,05$ & 0,2 \\
Distrito Federal & $-0,7331$ & $-7,55$ & $*$ & 0,1190 & 1,44 & 15,1 \\
\hline Coeficiente $c$ & \multicolumn{7}{c}{0,745} & 57.919 \\
№ de observações & 0,640 & & & \\
\hline
\end{tabular}

(1) Probabilidade caudal do teste da hipótese de nulidade do parâmetro. O asterisco assinala os casos em que essa probabilidade é inferior a $0,1 \%$.

Para avaliar os diversos determinantes do nível de insegurança alimentar nos domićlios brasileiros, foram obtidas as equações apresentadas na Tabela 9. Foram incorporadas as seguintes varáveis explanatórias:

a) Escolaridade ou anos de estudo da pessoa de referência da principal Unidade de Consumo do domicílio, com valores de 0 a 16, atribuindo-se valor 16 para os com 16 ou mais anos de estudo;

b) A proporção da renda total do domicílio proveniente de aposentadorias pagas pelo INSS;

c) A proporção da renda total do domicílio proveniente de aposentadorias de funcionários públicos estatutários;

d) A proporção da renda total do domicílio correspondente à renda não monetária;

e) Variável binária $(0,1)$ que assume valor 1 se a renda do domicílio inclui BPC (Benefício de Prestação Continuada);

f) Variável binária que assume valor 1 se a renda do domicilio inclui transferência do Programa BolsaFamília;

g) Variável binária com valor 1 se há membro com menos de 18 anos de idade.
Na Tabela 9 não se mostra o nível de significância dos testes $t$ ou a probabilidade caudal do teste da hipótese de nulidade do parâmetro (valor-p). Mas a distribuição de $t$ com número de graus de liberdade elevado se confunde com a distribuição de $Z$ (normal com média zero e variância igual a 1), podendo se verificar, então, que, para um teste bilateral, um valor absoluto $|t|$ igual ou maior que 2 é significativo a $5 \%$, $|t| \geq 2,6$ é significativo a $1 \%,|t| \geq 3,3$ é significativo a $0,1 \%,|t| \geq 3,9$ é significativo a $0,01 \%$ e $|t| \geq 4,5$ é significativo a $0,001 \%$. Note-se que os valores absolutos de $t$ para o efeito da RDPC são superiores a 18 nas três equações e os correspondentes valores para o efeito da escolaridade da pessoa de referência são superiores a 8 , mostrando a força ou importância dessas variáveis como condicionantes da insegurança alimentar. O crescimento da renda e da escolaridade são os meios básicos de reduzir a insegurança alimentar. Maior escolaridade permite obter renda mais elevada, mas mesmo depois de controlado o efeito da renda, os dados indicam que maior escolaridade contribui para reduzir a insegurança alimentar. 
Tabela 9. Modelo de lógite para a probabilidade de um domicílio ter insegurança alimentar de qualquer grau, insegurança alimentar moderada ou grave e apenas insegurança alimentar grave, Brasil, 2017-2018

\begin{tabular}{|c|c|c|c|c|c|c|}
\hline \multirow{2}{*}{ Variável } & \multicolumn{2}{|c|}{ Insegurança alimentar } & \multicolumn{2}{|c|}{$\begin{array}{l}\text { Insegurança moderada ou } \\
\text { grave }\end{array}$} & \multicolumn{2}{|c|}{ Insegurança grave } \\
\hline & $\begin{array}{c}\text { Estimativa do } \\
\text { parâmetro }\end{array}$ & $\begin{array}{c}\text { Teste } \\
t\end{array}$ & $\begin{array}{c}\text { Estimativa do } \\
\text { parâmetro }\end{array}$ & $\begin{array}{c}\text { Teste } \\
t\end{array}$ & $\begin{array}{l}\text { Estimativa } \\
\text { do } \\
\text { parâmetro }\end{array}$ & $\begin{array}{c}\text { Teste } \\
t\end{array}$ \\
\hline Constante & 5,2884 & 27,27 & 3,7973 & 18,93 & 2,2426 & 8,39 \\
\hline $\log (\mathrm{RDPC})$ & $-0,7834$ & $-29,34$ & $-0,7464$ & $-26,86$ & $-0,6887$ & $-18,78$ \\
\hline Escolaridade & $-0,0287$ & $-8,23$ & $-0,0490$ & $-10,72$ & $-0,0685$ & $-10,01$ \\
\hline Norte & 0,2687 & 5,15 & 0,3696 & 6,37 & 0,5020 & 6,33 \\
\hline Sudeste excl. SP & $-0,3997$ & $-8,56$ & $-0,4182$ & $-7,29$ & $-0,2808$ & $-2,84$ \\
\hline São Paulo & $-0,1864$ & $-3,40$ & $-0,4332$ & $-5,43$ & $-0,3430$ & $-2,82$ \\
\hline Sul & $-0,8195$ & $-15,2$ & $-0,8527$ & $-10,12$ & $-0,4704$ & $-4,19$ \\
\hline C. Oeste excl. DF & $-0,1862$ & $-3,41$ & $-0,1239$ & $-1,62$ & 0,0999 & 0,99 \\
\hline Distrito Federal & 0,1475 & 1,79 & 0,2799 & 2,63 & 0,6069 & 3,93 \\
\hline Rural & $-0,2713$ & $-6,61$ & $-0,2841$ & $-5,50$ & $-0,3139$ & $-4,54$ \\
\hline Proporção INSS & $-0,2925$ & $-5,02$ & $-0,2445$ & $-3,14$ & $-0,5106$ & $-4,11$ \\
\hline Proporção apo. pública & $-0,4426$ & $-3,70$ & $-0,6469$ & $-2,84$ & $-0,3286$ & $-0,78$ \\
\hline $\begin{array}{l}\text { Proporção de renda não } \\
\text { monetária }\end{array}$ & 0,8278 & 9,09 & 1,2101 & 11,18 & 1,3276 & 9,35 \\
\hline Recebe BPC & 0,3136 & 4,64 & 0,3128 & 3,88 & 0,2554 & 2,42 \\
\hline Recebe Bolsa Família & 0,4501 & 10,61 & 0,5980 & 12,26 & 0,6034 & 7,72 \\
\hline Tem Menor de 18 & 0,0405 & 1,24 & $-0,6721$ & $-15,74$ & $-0,7441$ & $-12,21$ \\
\hline Coeficiente $c$ & & 0,754 & & 0,774 & & 0,784 \\
\hline № de observações & & 57.919 & & 57.919 & & 57.919 \\
\hline
\end{tabular}

Mesmo depois de incluir vários outros controles, os sinais dos efeitos da localização regional permanecem iguais aos observados na segunda equação da Tabela 8. Em comparação com o Nordeste, fixado o valor de todas as demais variáveis explanatórias listadas na Tabela 9, a insegurança alimentar tende a ser maior no Norte e no Distrito Federal e tende a ser menor nas demais regiões, especialmente no Sul. A estimativa de efeito positivo da localização no Distrito Federal está associada ao fato de a RDPC média nessa Unidade da Federação ser muito mais alta do que no Nordeste e isso implicar em uma estimativa de insegurança alimentar mais baixa do que a observada. Essa discrepância se deve, pelo menos em parte, à elevada desigualdade da distribuição da renda no Distrito Federal[8].
O efeito da localização rural é negativo e significativo nas três equações apresentadas na Tabela 9 , mostrando que, depois de controlados os efeitos das demais variáveis explanatórias, estar em área rural reduz a probabilidade de um domicílio ter insegurança alimentar.

Quanto mais alta a participação das aposentadorias e pensões pagas pelo poder público na renda, menor tende a ser a insegurança alimentar. Isso pode ser devido, pelo menos em parte, à relativa estabilidade dessa parcela da renda. Por outro lado, uma maior participação das rendas não monetárias (que inclui o valor da produção para auto consumo na agricultura familiar) na renda total está associada a mais insegurança alimentar. 
A presença de pessoa com menos de 18 anos (o que inclui presença de crianças) não mostrou efeito estatisticamente significativo sobre a probabilidade de insegurança alimentar de qualquer grau, mas mostra efeito negativo e fortemente significativo sobre a probabilidade de insegurança alimentar moderada ou grave ou, especificamente, sobre a insegurança grave, confirmando resultados obtidos na análise dos dados da PNAD de 2013[4].

Nas três equações da Tabela 9, o fato de o domicílio incluir beneficiário de BPC ou Bolsa-Família, depois de controlado o efeito das demais variáveis, está associado a um aumento estatisticamente significativo da probabilidade de insegurança alimentar. Mas estaria totalmente errado concluir que a análise estatística está indicando que esses benefícios aumentam a insegurança alimentar. Eles representam acréscimos na renda que reduzem a insegurança alimentar. Vamos admitir, por exemplo, que o benefício triplique a RDPC de uma pessoa. Isso significa um acréscimo de 1,099 no seu logaritmo, pois

$\log (3 \cdot \mathrm{RDPC})=\log (\mathrm{RDPC})+\log (3)=\log (\mathrm{RDPC})+1,099$

Considerando o coeficiente de $\log (\mathrm{RDPC})$ na equação para insegurança alimentar grave, isso implica em reduzir o lógite da insegurança grave em 0,6887 $\times$ $1,099=0,7569$, o que é um valor absoluto maior do que os dos coeficientes da mesma equação associados à presença de beneficiários de BPC ou de Bolsa-Família no domicilio. Para essa pessoa hipotética a equação indica que o benefício reduz a insegurança alimentar grave. Como entender que, fixada a renda, um beneficiário de BPC ou Bolsa-Família tenda a apresentar insegurança alimentar maior do que pessoa não beneficiária? A obtenção desses benefícios depende de a pessoa tomar a iniciativa de reivindicá-los; deve haver, portanto um certo tipo de "viés de seleção" em favor de pessoas mais ativas na demanda por seus direitos. Tais pessoas tendem a se manifestar mais intensamente sobre situações que consideram insatisfatórias ou injustas, o que, dado o caráter parcialmente subjetivo da EBIA, faz com que sejam mais facilmente classificadas nos vários graus de insegurança alimentar. Mas isso é apenas uma tentativa de explicação. $\mathrm{O}$ esclarecimento do fenômeno exige pesquisa mais aprofundada, com informações mais específicas.
Procurou-se, nesta seção, avaliar a relação da insegurança alimentar com seus principais determinantes ou condicionantes. É obvio que a insegurança alimentar, por sua vez, deve se refletir em uma dieta menos variada e nutricionalmente mais pobre. O consumo de alimentos com elasticidade-renda elevada (ver Vaz e Hoffmann, 2020[10]) deve diminuir à medida que aumenta o grau de insegurança alimentar.

\section{CONCLUSÃO}

Estudos anteriores e a análise dos dados da POF 2017-2018 para as 27 Unidades da Federação mostram a forte relação da insegurança alimentar com o nível de renda e, especialmente, com medidas de pobreza. A comparação com a evolução da pobreza de 2004 a 2017-2018 mostra que o crescimento intenso da insegurança alimentar de 2013 a 2017-2018 (especialmente da insegurança alimentar leve) se deve, em parte, a certo grau de subjetividade da EBIA. As respostas às 14 perguntas, que são a base para a determinação do grau de insegurança alimentar de um domicílio certamente são afetadas de maneira especial por uma redução da renda depois de mais de uma década de redução da pobreza. A evolução da insegurança alimentar grave se mostra mais diretamente associada à evolução da pobreza.

A análise dos dados de 57.919 domicílios da amostra da POF 2017-2018 permitiu confirmar a associação da insegurança alimentar com diversas variáveis. Destacamos o efeito da renda e da escolaridade. Mas há relações para as quais pudemos apenas especular sobre possíveis explicações. Apontar dúvidas faz parte do trabalho do pesquisador. Aliás, há muito o que estudar. Pela primeira vez, em 2017-2018, a EBIA é aplicada em uma Pesquisa de Orçamentos Familiares (POF) no Brasil. Isso abre um amplo leque de pesquisas para relacionar a compra e ingestão de alimentos com as medidas de segurança ou insegurança alimentar.

\section{REFERÊNCIAS}

[1] IBGE. Pesquisa de orçamentos familiares 2017-2018: análise da segurança alimentar no Brasil. Rio de Janeiro: IBGE - Coordenação de Trabalho e Rendimento; 2020. 
[2] Hoffmann R. Determinantes da insegurança alimentar no Brasil: análise dos dados da PNAD de 2004. Segur. Aliment. Nutr. 2008; 15(1):49-61.

[3] Hoffmann R. Determinantes da insegurança alimentar no Brasil em 2004 e 2009. Segur. Aliment. Nutr. 2013; 20(2):219235.

[4] Hoffmann R. Brasil, 2013: mais segurança alimentar. Segur. Aliment. Nutr. 2014; 21(2):422-438.

[5] Hoffmann R. Distribuição da renda domiciliar per capita no Brasil, 2012 a 2019 e 1995 a 2015. Texto para Discussão n. 59 do IEPE/Casa das Garças. 2020 mai.

[6] Hoffmann R. Desigualdade de renda no Brasil, 1995 a 2019: diversas distribuições e o impacto do desemprego. Revista Brasileira de Economia Social e do Trabalho. 2020; 2:1-27.

[7] IBGE. Pesquisa de orçamentos familiares 2017-2018: análise do consumo alimentar pessoal no Brasil. Rio de Janeiro: IBGE - Coordenação de Trabalho e Rendimento; 2020.

[8] Hoffmann R, Vaz DV. Mensurando a desigualdade no Brasil: evidências a partir da renda e dos gastos das famílias. Texto para Discussão n. 63 do IEPE/Casa das Garças. 2020 jun.

[9] IBGE. Pesquisa de orçamentos familiares 2017-2018: primeiros resultados. Rio de Janeiro: IBGE - Coordenação de Trabalho e Rendimento; 2019.

[10] Vaz DV, Hoffmann R. Elasticidade-renda e concentração das despesas com alimentos no Brasil: uma análise dos dados das POF de 2002-2003, 2008-2009 e 201720178. Revista de Economia. 2020; 41(75):282-310. 